\title{
1 Molecular processes of transgenerational acclimation to a warming ocean
}

2 Heather D. Veilleux ${ }^{1,2^{*}}$, Taewoo Ryu ${ }^{3^{*}}$, Jennifer M. Donelson ${ }^{4,2}$, Lynne van Herwerden ${ }^{2,5}$,

3 Loqmane Seridi $^{3}$, Yanal Ghosheh ${ }^{3}$, Michael L. Berumen ${ }^{6}$, William Leggat ${ }^{1,7}$, Timothy Ravasi ${ }^{3 \mp}$,

$4 \quad$ Philip L. Munday ${ }^{1,2 \mp}$

$5{ }^{1}$ ARC Centre of Excellence for Coral Reef Studies, James Cook University, Townsville QLD 6 4811, Australia

$7 \quad{ }^{2}$ College of Marine and Environmental Sciences, James Cook University, Townsville QLD 8 4811, Australia

$9{ }^{3}$ KAUST Environmental Epigenetic Program, Division of Applied Mathematics and Computer 10 sciences, King Abdullah University of Science and Technology, 23955, Thuwal, Kingdom of 11 Saudi Arabia

$12{ }^{4}$ School of Life Sciences, University of Technology Sydney, P.O. Box 123, Broadway, NSW 13 2007, Australia

$14{ }^{5}$ Centre for Tropical Fisheries and Aquaculture, James Cook University, Townsville QLD 15 4811, Australia

$16{ }^{6}$ Red Sea Research Center, King Abdullah University of Science and Technology, Thuwal, 1723955, Kingdom of Saudi Arabia

$18{ }^{7}$ College of Medical, Veterinary and Biomedical Sciences, James Cook University, Townsville, 19 QLD, 4811, Australia

$20 *$ These authors contributed equally to this work.

21 干Email: timothy.ravasi@kaust.edu.sa; philip.munday@jcu.edu.au. 
22 Some animals have the remarkable capacity to acclimate across generations to projected

23 future climate change ${ }^{1-4}$; however, the underlying molecular processes are unknown. We

24 sequenced and assembled de novo transcriptomes of adult tropical reef fish exposed developmentally or transgenerationally to projected future ocean temperatures and correlated the resulting expression profiles with acclimated metabolic traits from the same fish. We identified 69 contigs representing 53 key genes involved in thermal acclimation of aerobic capacity. Metabolic genes were among the most upregulated transgenerationally suggesting shifts in energy production for maintaining performance at elevated temperatures. Furthermore, immune and stress responsive genes were upregulated transgenerationally, indicating a new compliment of genes allowing the second generation of fish to better cope with elevated temperatures. Other differentially expressed genes were involved with tissue development and transcriptional regulation. and transgenerational treatments. Heat shock protein genes were surprisingly unresponsive, indicating that short-term heat stress responses may not be a good indicator of long-term acclimation capacity. Our results are the first to reveal the molecular processes that may enable marine fishes to adjust to a future warmer environment over multiple generations.

Over the next century, rising ocean temperatures due to climate change will pose a serious threat to the survival of many aquatic species. To persist, populations will either need to

42 shift their geographic distributions ${ }^{5}$ or adapt through genetic evolution or phenotypic

43 plasticity ${ }^{6-8}$. Of particular concern for marine species, is that rising temperatures will reduce 
44 the capacity for oxygen supply and delivery ${ }^{9,10}$, limiting activities essential to survival and

45 individual fitness. Reduced aerobic scope (the capacity for oxygen uptake above resting 46 metabolic rate) at higher temperatures can affect vital functions such as growth, swimming 47 performance, reproduction and competitive ability ${ }^{10-14}$. In reef fishes, aerobic scope declines 48 at temperatures just a few degrees above the summer average, within the range projected 49 to occur as a result of climate change ${ }^{9,12,15}$. However, aerobic capacity can be fully restored 50 transgenerationally, when parents and their offspring both experience the same elevated 51 temperatures (transgenerational acclimation) ${ }^{1}$. Understanding the molecular processes that 52 make this transgenerational plasticity possible is important for assessing the performance of 53 marine organisms and sustainability of their populations in a rapidly warming ocean.

54 We used a multi-generational rearing experiment to identify the molecular pathways 55 associated with transgenerational thermal acclimation of metabolic traits in a common reef 56 fish, Acanthochromis polyacanthus. Second generation fish were reared developmentally 57 (from hatching to adulthood) and transgenerationally (two generations) at two elevated temperatures $\left(+1.5\right.$ and $\left.+3.0^{\circ} \mathrm{C}\right)$ and in control conditions $\left(+0.0^{\circ} \mathrm{C}\right.$; Fig. 1a). The full 59 transcriptome of four to five adult fish from each of the five treatments (Fig. 1a) was sequenced and expression data correlated to standardised metabolic traits from the same 61 fish: routine metabolic rate (RMR), maximum metabolic rate (MMR) and net aerobic scope 62 (MMR - RMR; NAS; Supplementary Methods). As observed in previous studies ${ }^{1}$, developmental exposure to elevated temperatures from just after hatching into adulthood 64 led to a reduction in aerobic scope (Fig. 1b). However, when both parents and offspring 65 were exposed to elevated temperatures, complete restoration of aerobic scope was 66 achieved (Fig. 1b). Of 89,543 assembled contiguous sequences (contigs), 165 had significant 
67 differential expression (adjusted $\mathrm{P}<0.05$ ) in at least one of the treatment comparisons

68 (transgenerational and developmental treatments vs. control; transgenerational vs.

69 developmental treatments). One hundred and sixty of the differentially expressed contigs

70 had BLASTN and/or BLASTP ${ }^{16}$ sequence matches with e-values less than $10^{-10}$, of which 69

71 had expression that was significantly correlated to at least one of the standardised

72 metabolic measures (RMR, MMR, and NAS). Comparing transgenerational and

73 developmental treatments at the same temperatures enabled us to distinguish patterns of

74 gene expression due to transgenerational effects, compared with effects of within-

75 generation exposure to elevated temperatures.

76 The 69 differentially expressed and correlated contigs represent 52 genes that are

77 associated with transgenerational thermal acclimation. These genes are involved in a variety

78 of cellular processes such as metabolism, transport, immune and stress responses, growth

79 and development, cell cycle, cell organisation, and transcriptional regulation (Fig. 2;

80 Supplementary Table 1). The expression profiles of these contigs separated into three

81 distinct groups, with the first and largest group (Fig. 2a; 46 contigs) containing contigs with

82 expression that primarily correlated to the acclimating phenotypic trait, NAS (78\%).

83 Metabolism is the major function associated with genes in this group (lipid, protein, and

84 carbohydrate metabolism; nine, nine, and five contigs each, respectively), including $79 \%$ of

85 the most highly upregulated contigs transgenerationally relative to controls $(\geq 1.5 \log 2$ fold

86 change; Supplementary Table 1). During thermal stress, the composition of lipid

87 membranes is altered (homeoviscous adaptation) ${ }^{17}$ and there are changes in lipid use ${ }^{18}$ and

88 expression of the fatty acid pathway ${ }^{19}$. Of the nine contigs associated with lipid metabolism,

89 six were strongly upregulated in transgenerational treatments (representing four genes: 
90 acsl5, adtrp, apoEb, and pdzk1). ApoE has a major role in triglyceride and cholesterol

91 homeostasis, suggesting that transgenerational upregulation of lipid metabolism may be

92 critical for improved aerobic scope. ApoE and other apolipoproteins are also upregulated

93 after short-term thermal challenge in fish ${ }^{20,21}$, suggesting a link between short-term thermal

94 stress and long-term thermal acclimation of aerobic capacity. Many of the metabolic genes

95 in the first group (Fig. 2a) are involved in catabolism and digestion (Supplementary Table 1),

96 suggesting their augmented expression provides increased energy for aerobic performance

97 in transgenerational fish (Supplementary Table 1). Supporting this hypothesis, 11 contigs are

98 involved in the cellular transport of ions, solutes, amino acids, lipids, and carbohydrates,

99 possibly as a result of increased substrate digestion. Our results suggest that there is

100 transgenerational regulation of lipid, protein, and carbohydrate metabolism and that each

101 may be critical for increased energy use associated with acclimation of aerobic scope across

102 generations.

103 In addition to metabolic responses, sixteen contigs with putative functions associated with

104 immune responses and inflammation, apoptosis, homeostasis, and stress were significantly

105 upregulated during transgenerational thermal acclimation (Fig. 2a). Immune responses can

106 be maternally imprinted in fish ${ }^{22}$, potentially by transferring maternal idiotypic networks to

107 juveniles at a critical stage ${ }^{23}$. Such imprinting, we hypothesise, would then be augmented

108 throughout development to establish an immune response better suited for survival under

109 thermal stress. As chronic stress can suppress immune function and lead to increased

110 susceptibility to disease and pathogens ${ }^{24}$, the transgenerational augmentation of five

111 putative immune-related contigs (gimap8, xpnpep2, mep1b, and natterin3) may represent 
112 new baseline levels of immune-related genes to protect against elevated temperatures 113 experienced across generations.

114 The second major group of genes (Fig. 2b) is comprised of 12 contigs, all of which had 115 expression that was negatively correlated to standardised RMR. RMR was lower in fish 116 exposed transgenerationally to $+3.0^{\circ} \mathrm{C}$ compared with controls (Supplementary Fig. 1). The 117 high proportion of contigs in this group with putative function in organ development (two 118 contigs; ppdpfa and ptf1a) and endothelial cell proliferation (four contigs; nlrp14 and timp2) suggests that lower metabolic costs enabled these cellular processes to function at a higher 120 level in transgenerationally acclimated fish, which is consistent with acclimation of growth 121 rates in fish exposed transgenerationally to elevated temperatures ${ }^{3,4}$. In addition, this group 122 contains five contigs related to transcriptional regulation (three genes: rorb, ptf1a, and 123 rps27), two of which enhance expression of genes involved in organogenesis (rorb, ptf1a). 124 The third gene, rps27, is a nuclear protein induced upon DNA damage ${ }^{25}$. Therefore, 125 increased transgenerational expression and negative correlation to standardised RMR suggest rps27 plays a role in maintaining DNA integrity after transgenerational exposure to 127 elevated temperatures to restore routine metabolic function.

128 While the first two groups in the heatmap (Fig. 2a, Fig. 2b) contained contigs with 129 expression that was significantly elevated transgenerationally, the third group (Fig. 2c) contained 11 contigs (16\% of total) with expression that was downregulated 131 transgenerationally. The majority of these contigs had expression that positively correlated 132 to standardised RMR (64\%; seven contigs) and largely matched genes with functions related 133 to stress, homeostasis, and immune responses (Supplementary Table 1). As many other 134 stress and immune-related genes were upregulated transgenerationally in the first two 
groups, the downregulated genes with these functions in the final group suggests their expression was reduced in favour of other more beneficial genes for transgenerational 137 acclimation.

138 The heatmap indicates that many contigs had higher differential expression in 139 transgenerational compared with developmental treatments (Fig. 2); however, only three 140 were statistically significant: cytochrome p450 2j2 (cyp2j2), ribosomal protein large P1 141 (rplp1), and an uncharacterised gene (Supplementary Table 1). Cyp2j2 is associated with 142 epoxidation of arachidonic $\mathrm{acid}^{26}$, of which the primary products formed, 143 epoxyeicosatrienoic acids, are involved in a variety of processes such as vasodilation, anti144 inflammation, and cytoprotection. For example, cyp2j2 appears to play a cytoprotective role 145 in animals exposed to hypoxia ${ }^{27}$ and high-fat diets ${ }^{28}$. Thus, we hypothesize that increased 146 transgenerational cyp2j2 expression may play an important cytoprotective role, allowing 147 proper cellular function after transgenerational but not developmental exposure to elevated 148 temperatures. Rplp1 plays a key role in the elongation step of protein synthesis. Therefore, 149 rplp1 may be required in developmental treatments to increase protein translation due to a 150 higher rate of protein degradation during thermal stress, but is no longer required 151 transgenerationally due to the aforementioned increases in cytoprotective gene expression. 152 Importantly, there was only one contig (btn1a1) that was significantly differentially 153 expressed in developmental but not transgenerational treatments (Supplementary Table 1).

154 This suggests that there is not a different suite of genes and cellular processed engaged 155 during developmental exposure to elevated temperatures compared with transgenerational 156 acclimation. 
157 A commonly used molecular measure of thermal stress has been to examine molecular 158 chaperone expression, specifically heat shock proteins (HSPs). Some HSPs are constitutively 159 expressed and are involved in nascent polypeptide folding, while others are expressed to 160 help refold proteins that unfolded due to various stressors ${ }^{29}$. We found no HSP genes with 161 significantly altered expression in developmental or transgenerational A. polyacanthus. Of 162 all 160 significantly differentially expressed contigs identified in this study, including contigs 163 with expression that did and did not correlate to metabolic traits (Supplementary Fig. 2), 164 only one matched a gene with putative chaperone function: eukaryotic translation 165 elongation factor 1a (eef1a). This gene has been shown to protect aminoacyl-tRNA 166 synthetases from denaturation in mammals ${ }^{30}$, and may therefore have a more specific role 167 in maintaining the integrity of transgenerational protein synthesis in our study. While 168 contigs with matches for many HSPs were found within the A. polyacanthus transcriptome, 169 none were significantly differentially expressed among the five treatments (Fig. 3; adjusted $170 P>0.7)$. Therefore, the lack of differential HSP gene expression and limited chaperone 171 activity suggests that other genes outlined in this study are better indicators of 172 transgenerational thermal acclimation, at least in A. polyacanthus. While HSPs may be good 173 indicators of acute thermal stress ${ }^{29,31}$, our results suggest they may not be good indicators 174 of the capacity for long-term thermal acclimation to predicted temperatures under climate 175 change.

176 Acclimation of aerobic scope within two generations ${ }^{1}$ suggests epigenetic inheritance is 177 involved. Future research into epigenetic mechanisms and their effect on genes identified in 178 this study will be useful to improve our understanding of adaptive responses to rapid 179 environmental change. In this study we identified key genes and processes involved in 
transgenerational thermal acclimation, including genes involved in enhanced fatty acid oxidation, protein and carbohydrate metabolism, and changes in genes involved in cytoprotection, immunity, organogenesis, and cellular organisation. The plasticity of these genes and their strong correlation to known acclimating phenotypic traits suggests they may be critical in aiding reef fishes, and possibly other marine organisms to survive in a warmer future environment.

\section{Methods Summary}

Two generations of $A$. polyacanthus were reared as per Donelson et al. ${ }^{1}$ and livers were dissected from control, developmental, and transgenerational temperature treated adults. mRNA was extracted and cDNA libraries constructed for transcriptome sequencing on an Illumina HiSeq 2000 platform analyser. Reads were assembled de novo using Trans-ABySS ${ }^{32}$. Normalised transcriptome expression data was validated by quantitative real-time PCR for eight genes, consisting of both up and downregulated genes (Supplementary Fig. 3, Supplementary Table 2). Standardised metabolic measurements (RMR, MMR and NAS; Supplementary Methods) for each individual were correlated to the normalised and variance stabilised expression data for each contig, of which only those with a significant ( $P$ $\leq 0.05)$ correlation were selected.

\section{Online Content}

Methods, Supplementary tables, and additional Supplementary display items are available in the online version of the paper; references unique to these sections appear only in the online paper.

\section{References}


1. Donelson, J. M., Munday, P. L., McCormick, M. I. \& Pitcher, C. R. Rapid transgenerational acclimation of a tropical reef fish to climate change. Nature Clim. Change 2, 30-32, (2012).

2. Miller, G. M., Watson, S.-A., Donelson, J. M., McCormick, M. I. \& Munday, P. L. Parental environment mediates impacts of increased carbon dioxide on a coral reef fish. Nature Clim. Change 2, 858-861, (2012).

3. Salinas, S. \& Munch, S. B. Thermal legacies: transgenerational effects of temperature on growth in a vertebrate. Ecology Letters 15, 159-163, (2012).

4. Shama, L. N. S., Strobel, A., Mark, F. C. \& Wegner, K. M. Transgenerational plasticity in marine sticklebacks: maternal effects mediate impacts of a warming ocean. Functional Ecology, doi:10.1111/1365-2435.12280, (2014).

5. Poloczanska, E. S. et al. Global imprint of climate change on marine life. Nature Clim. Change 3, 919-925, (2013).

6. Munday, P. L., Warner, R. R., Monro, K., Pandolfi, J. M. \& Marshall, D. J. Predicting evolutionary responses to climate change in the sea. Ecol. Lett. 16, 1488-1500, (2013).

7. Palumbi, S. R., Barshis, D. J., Traylor-Knowles, N. \& Bay, R. A. Mechanisms of reef coral resistance to future climate change. Science 344, 895-898, (2014).

8. Munoz, N. J., Farrell, A. P., Heath, J. W., \& Neff, B. D. Adaptive potential of a Pacific salmon challenged by climate change. Nature Clim. Change 5, 163-166, (2015).

9. Nilsson, G. E., Crawley, N., Lunde, I. G. \& Munday, P. L. Elevated temperature reduces the respiratory scope of coral reef fishes. Glob. Change Biol. 15, 1405-1412, (2009).

10. Portner, H. O. \& Farrell, A. P. Physiology and climate change. Science 322, 690-692, (2008).

11. Portner, H. O. \& Knust, R. Climate change affects marine fishes through the oxygen limitation of thermal tolerance. Science 315, 95-97, (2007).

12. Johansen, J. L. \& Jones, G. P. Increasing ocean temperature reduces the metabolic performance and swimming ability of coral reef damselfishes. Glob. Change Biol. 17, 2971-2979, (2011).

13. Eliason, E. J. et al. Differences in thermal tolerance among sockeye salmon populations. Science 332, 109-112, (2011).

14. Killen, S. S. et al. Aerobic scope predicts dominance during early life in a tropical damselfish. Funct. Ecol., doi: 10.1111/1365-2435.12296, (2014).

15. Rummer, J. L. et al. Life on the edge: thermal optima for aerobic scope of equatorial reef fishes are close to current day temperatures. Glob. Change Biol. 20, 1055-1066, (2014).

16. Altschul, S. F. et al. Gapped BLAST and PSI-BLAST: a new generation of protein database search programs. Nucleic Acids Res. 25, 3389-3402, (1997).

17. Hazel, J. R. Thermal adaptation in biological membranes: is homeoviscous adaptation the explanation? Annu. Rev. Physiol. 57, 19-42, (1995).

18. Kieffer, J. D., Alsop, D. \& Wood, C. M. A respirometric analysis of fuel use during aerobic swimming at different temperatures in rainbow trout (Oncorhynchus mykiss). J. Exp. Biol. 201 3123-3133, (1998).

19. McClelland, G. B. Fat to the fire: the regulation of lipid oxidation with exercise and environmental stress. Comp. Biochem. Phys. B. 139, 443-460, (2004).

20. Kassahn, K. S., Crozier, R. H., Ward, A. C., Stone, G. \& Caley, J. M. From transcriptome to biological function: environmental stress in an ectothermic vertebrate, the coral reef fish Pomacentrus polyacanthus. BMC Genom. 8, 358, (2007). 
21. Podrabsky, J. E. \& \& Somero, G. N. Changes in gene expression associated with acclimation to constant temperatures and fluctuating daily temperatures in an annual killifish Austrofundulus limnaeus. J. Exp. Biol. 207, 2237-2254 (2004).

22. Bly, J. E., Grimm, A. S. \& Morris, I. G. Transfer of passive immunity from mother to young in a teleost fish: haemagglutinating activity in the serum and eggs of plaice, Pleuronectes platessa L. Comp. Biochem. Phys. A. 84, 309-313, (1986).

23. Lemke, H. \& Lange, $H$. Is there a maternally induced immunological imprinting phase a la Konrad Lorenz? Scand. J. Immunol. 50, 348-354, (1999).

24. Bonga, S. E. W. The stress response in fish. Phsiol. Rev. 77, 591-625 (1997).

25. Xiong, X., He, H., Sun, Y. Ribosomal protein S27-like and S27 interplay with p53-MDM2 axis as a target, a substrate and a regulator. Oncogene 30, 1798-1811 (2011).

26. Zeldin, D. C. Epoxygenase Pathways of Arachidonic Acid Metabolism. J. Biol. Chem. 276, 36059-36062 (2001).

27. Yang, B. et al. Overexpression of Cytochrome P450 protects against hypoxiareoxygenation injury in cultured bovine aortic endothelial cells. J. Pharmacol. Exp. Ther. 60, 310-320 (2001).

28. Chen, G. et al. CYP2J2 overexpression attenuates nonalcoholic fatty liver disease induced by high-fat diet in mice. Am. J. Physiol. Endocrinol. Metab. 308, E97-E110 (2015).

29. Feder, M. E. \& Hofmann, G. E. Heat-shock proteins, molecular chaperones, and the stress response: evoluationary and ecological physiology. Annu. Rev. Physiol. 61, 243282 (1999).

30. Lukash, T. O., Turkivska, H. V., Negrutskii, B. S. \& El'skaya, A. V. Chaperone-like activity of mammalian elongation factor eEF1A: renaturation of aminoactyl-tRNA synthetases. Int. J. Biochem. Cell Biol. 36, 1341-1347 (2004).

31. Basu, N. et al. Heat shock protein genes and their functional significance in fish. Gene 295, 173-183 (2002).

32. Robertson, G. et al. De novo assembly and analysis of RNA-seq data. Nature Methods 7, 909-912, (2010).

201

202

203

204

205

206

207

\section{Acknowledgements}

This study was supported by the Australian Research Council (ARC) and the ARC Centre of Excellence for Coral Reef Studies (P.L.M. and W.L.), King Abdullah University of Science and Technology (T.Ra., M.L.B., T.Ry, L.S., and Y.G.), the Australian Coral Reef Society (H.D.V.), and a GBRMPA Science for Management Award (H.D.V.). This project was completed under JCU Ethics A1233 and A1415. We thank J.L. Rummer for comments on the manuscript and members of the Molecular Ecology and Evolution Laboratory (JCU), Marine and Aquaculture 
208 Research Facilities Unit (JCU), Integrative Systems Biology Laboratory (KAUST), and

209 Biosciences Core Laboratory (KAUST) for support and assistance.

\section{Author contributions}

211 J.M.D. and P.L.M. designed and managed the fish rearing experiments. J.M.D. performed 212 metabolism experiments. H.D.V. prepared samples for sequencing. T.Ry. assembled 213 transcriptome. T.Ry., T. Ra., L.S., and Y.G. analysed expression and assessed assembly 214 quality. H.D.V. performed qRT-PCR expression validation. H.D.V. analysed the data. H.D.V., 215 P.L.M., T.Ry., J.M.D., L.v.H., M.L.B., W.L., and T.Ra. wrote the paper and all authors read and 216 approved the manuscript.

\section{Additional information}

RNA-seq transcriptome sequences have been deposited in GenBank under BioProject ID PRJNA255544. The authors declare no competing financial interests. Supplementary information accompanies this paper on http://www.nature.com/nature. Reprints and permissions information is available online at http://www.nature.com/reprints.

222 Correspondence and request for material should be addressed to P.L.M and T.Ra.

\section{Figure Legends}

224 Figure 1 | Transgenerational experimental design and corresponding net aerobic scope measures. a, Experimental design tree showing the three temperature treatments $\left(+0.0^{\circ} \mathrm{C}\right.$, $+1.5{ }^{\circ} \mathrm{C}$ and $\left.+3.0{ }^{\circ} \mathrm{C}\right)$ at which three generations $\left(\mathrm{F}_{0}, \mathrm{~F}_{1}\right.$ and $\left.\mathrm{F}_{2}\right)$ of Acanthochromis polyacanthus were reared. Temperature treatments are colour coded and experimental

228 duration for each generation is shown in the vertical grey bars to the right. Fish in the $F_{2}$ 229 generation representing control, developmental and transgenerational temperature 
treatments are indicated by horizontal grey bars. b, Net aerobic scope (NAS) of fish in control, developmental and transgenerational $F_{2}$ treatments (mean \pm s.e.m.). Lower case

232 letters above bars indicate significant differences $(P<0.05)$ among treatments. Number of

233 fish used to measure NAS for each treatment is shown beneath the grey bars.

234 Figure 2 | Differentially expressed contigs, correlations to metabolic performance, and putative cellular function. Heatmap (left) of differentially expressed contigs (adjusted $\mathrm{P}<$ 0.05) from Acanthochromis polyacanthus, comparing $+1.5{ }^{\circ} \mathrm{C}$ and $+3.0{ }^{\circ} \mathrm{C}$ developmental 237 (devel.) and transgenerational (transgen.) treatments to control $\left(+0.0{ }^{\circ} \mathrm{C}\right)$. Based on expression patterns, contigs were separated into three groups $(a, b$, and $c)$. The associated cellular functions for each group are presented as pie charts (middle), with each contig represented by two functions with the exception of those that were uncharacterised. Numbers within pie chart sections represent the total number of contigs that correspond to that function. Venn diagrams (right) indicate the proportion of contigs with expression that positively (blue) or negatively (red) correlated to metabolic data (NAS - net aerobic scope, MMR - maximum metabolic rate, and RMR - routine metabolic rate). Purple text indicates negative NAS and positive RMR.

Figure 3 | Heat shock protein (HSP) contig expression pattern. Heatmap of HSP expression from Acanthochromis polyacanthus, comparing $+1.5^{\circ} \mathrm{C}$ and $+3.0^{\circ} \mathrm{C}$ developmental (devel.) and transgenerational (transgen.) treatments to control $\left(+0.0^{\circ} \mathrm{C}\right)$. There were no significant differences in expression (adjusted $\mathrm{P}<0.05$ ). Expression values correspond to the contig with the best match $\left(E\right.$-value $\left.<10^{-27}\right)$ to HSP genes within our transcriptome. 
$251 \quad$ Figure 1.

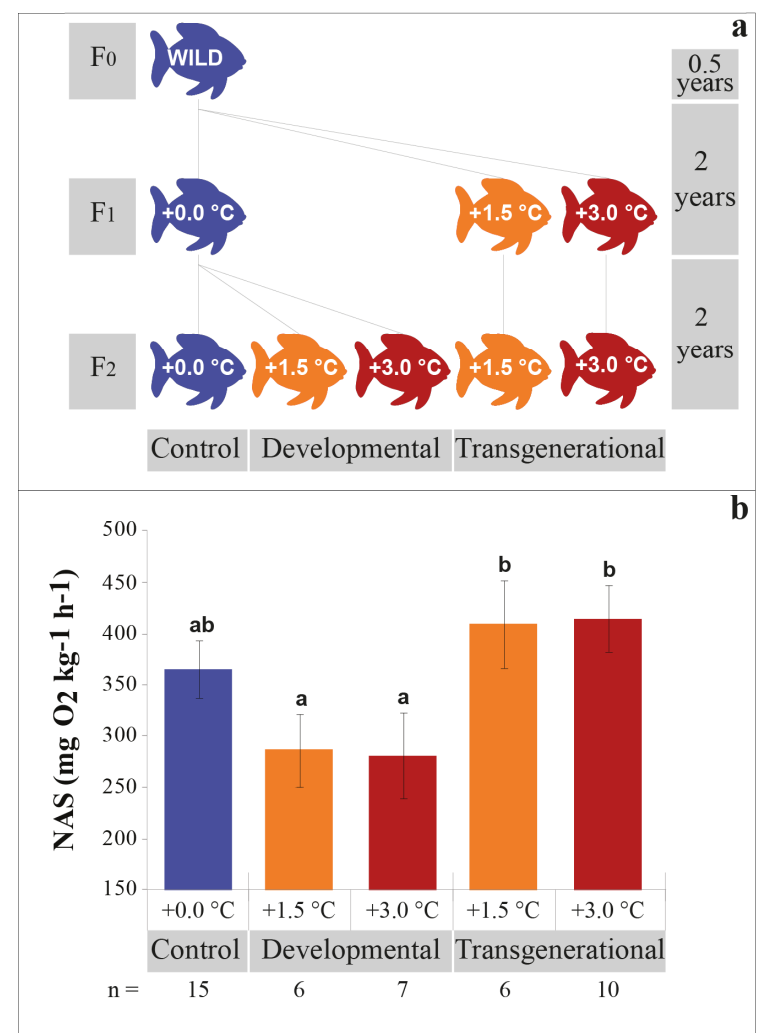


252 Figure 2. 

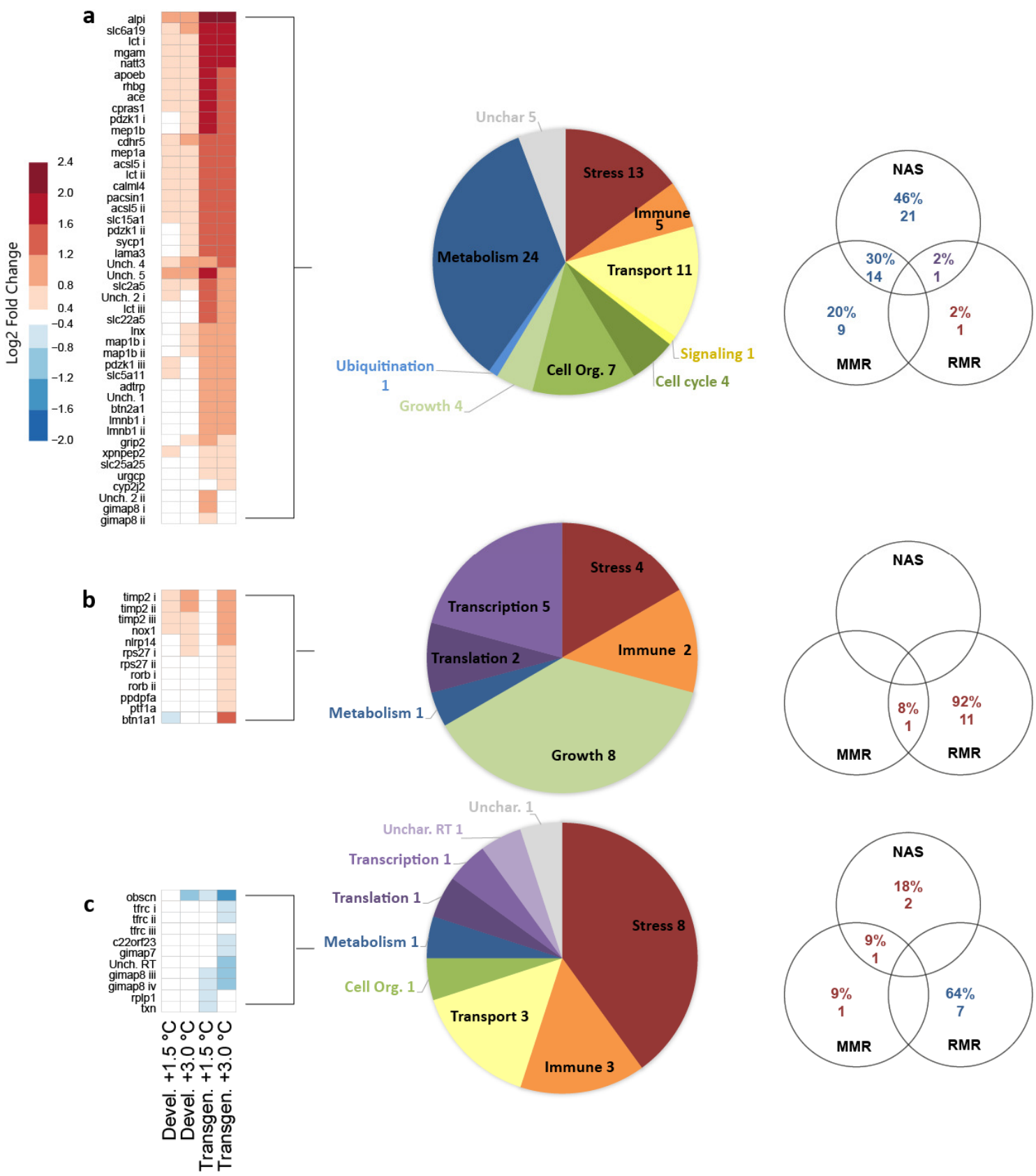
$253 \quad$ Figure 3.
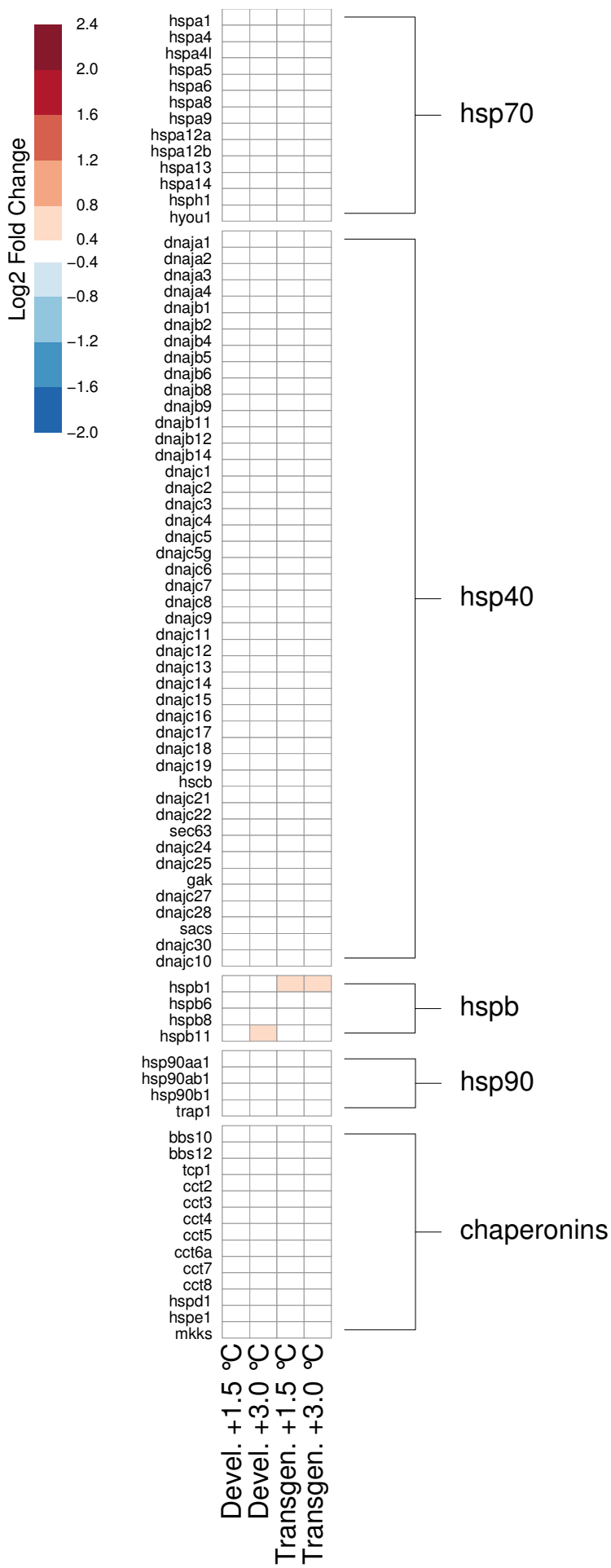